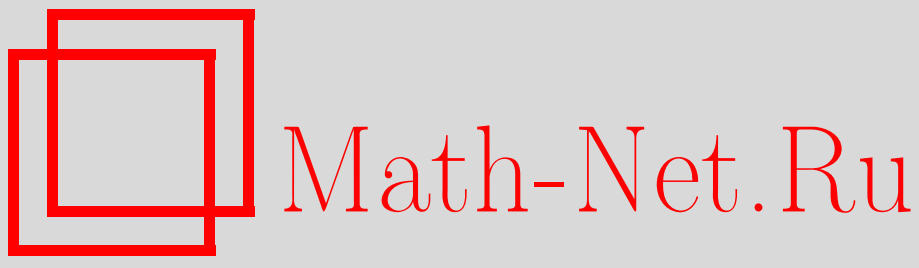

В. Е. Зотеев, Итерационный метод среднеквадратичного оценивания параметров корреляционной функции распределения случайного поля неупругой реологической деформации, Вестн. Сам. гос. техн. ун-та. Сер. Физ.-мат. науки, 2006, выпуск 42, 123-134

DOI: https://doi.org/10.14498/vsgtu423

Использование Общероссийского математического портала Math-Net.Ru подразумевает, что вы прочитали и согласны с пользовательским соглашением http://www . mathnet.ru/rus/agreement

Параметры загрузки:

IP: 54.81 .137 .203

26 апреля 2023 г., 09:44:47

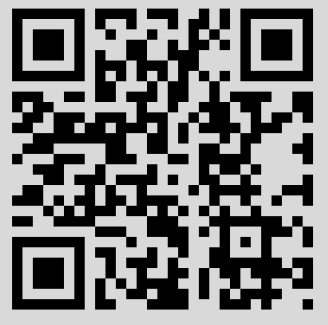




\section{ИТЕРАЦИОННЫЙ МЕТОД СРЕДНЕКВАДРАТИЧНОГО ОЦЕНИВАНИЯ ПАРАМЕТРОВ КОРРЕЛЯЦИОННОЙ ФУНКЦИИ РАСПРЕДЕЛЕНИЯ СЛУЧАЙНОГО ПОЛЯ НЕУПРУГОЙ РЕОЛОГИЧЕСКОЙ ДЕФОРМАЦИИ}

Рассматривается эффективный численный метод среднеквадратичного оиенивания параметров корреляционной функции распределения случайного поля неупругой реологической деформации. В основе метода лежит линейно параметрическая дискретная модель в форме стохастического разностного уравнения, коэффициенты которой известным образом связаны с параметрами корреляционной функции. Приводятся результаты численно-аналитических исследований сходимости метода и погрешности вычисления параметров корреляџионной функции. Данный метод может быть рекомендован для эффективного решения задач параметрической идентификачии различных по физической природе объектов, динамические прочессы в которых описываются затухаюшей по экспоненте синусоидой.

Рассматривается задача определения параметров корреляционной функции распределения случайного поля неупругой реологической деформации по экспериментальным данным [1]. Важность этой проблемы диктуется широким кругом методов решения краевых стохастических задач пластичности и ползучести, основанных на использовании аналитической аппроксимации корреляционной функции [2,3]. Особенностью исследуемого процесса является сильная зашумленность истинных значений корреляционной функции случайной аддитивной помехой в результатах наблюдений. Достоверное определение параметров корреляционной функции в таких условиях требует применения статистических методов обработки результатов эксперимента. Классические способы вычисления среднеквадратичных оценок параметров корреляционной функции приводят к численным итерационным методам решения нелинейной системы нормальных уравнений, эффективность которых сильно зависит от выбора начального приближения решения [4]. Как правило, применение таких способов в практике обработки результатов эксперимента вызывает серьезные затруднения.

В данной работе поставленная задача решается в формате современных информационных технологий. Принципиальным моментом для нового способа решения является разработка и применение линейно параметрической дискретной модели (ЛПДМ) корреляционной функции в форме стохастического разностного уравнения, коэффициенты которой известным образом связаны с параметрами корреляционной функции. При таком подходе задача определения параметров корреляционной функции случайного поля неупругой реологической деформации сводится к устойчивому среднеквадратичному оцениванию коэффициентов стохастического разностного уравнения [5].

Известно [1], что корреляционная функция распределения случайного поля неупругой реологической деформации описывается выражением вида

$$
\tilde{y}(x)=a_{0} \exp (-|\alpha| x)\left[\cos (|\beta| x)+\frac{\alpha}{\beta} \sin (|\beta| x)\right],
$$

где $\alpha, \beta$ и $a_{0}$ - параметры, подлежащие определению. В ходе эксперимента обычно формируются выборки небольших объемов $(N=20 \div 30)$, а результаты наблюдений $y_{k}=\tilde{y}\left(x_{k}\right)+\varepsilon_{k}$, $k=\overline{0, N-1}$, содержат случайную аддитивную составляющую $\varepsilon_{k}$, существенно искажающую теоретические значения корреляционной функции $\tilde{y}\left(x_{k}\right)$. Поэтому устойчивое и достоверное оценивание параметров $\alpha, \beta$ и $a_{0}$ является важнейшей проблемой при исследовании случайных полей неупругой реологической деформации.

В данной работе рассматривается новый метод определения параметров корреляционной функции распределения случайного поля неупругой реологической деформации, в основе которого лежит итерационная процедура среднеквадратичного оценивания коэффициентов ЛПДМ в форме стохастического разностного уравнения. Разработка этого метода включает решение следующих основных задач:

-построение линейно параметрической дискретной модели, связывающей последовательные отсчеты корреляционной функции, в форме стохастического разностного уравнения; 
- разработка алгоритма определения параметров корреляционной функции на основе среднеквадратичных оценок коэффициентов стохастического разностного уравнения;

-исследование эффективности итерационного метода среднеквадратичного оценивания коэффициентов ЛПДМ;

-оценка погрешности вычисления параметров корреляционной функции в процессе обработки результатов эксперимента.

Построение линейно параметрической дискретной модели корреляционной функции в форме стохастического разностного уравнения. Основой предлагаемого метода определения параметров корреляционной функции распределения случайного поля неупругой реологической деформации является линейно параметрическая дискретная модель. Следует различать линейно параметрическую дискретную модель в виде рекуррентной формулы, линейно связывающей некоторые комбинации дискретных значений исследуемой функциональной зависимости, и ЛПДМ в форме стохастического разностного уравнения, описывающего эту зависимость с учетом аддитивной случайной помехи в результатах наблюдений.

Рассмотрим построение ЛПДМ в виде рекуррентной формулы, связывающей три последовательных отсчета непрерывной функции $\tilde{y}(x)=a_{0} \exp (-\alpha x)\left[\cos (\beta x)+\frac{\alpha}{\beta} \sin (\beta x)\right]$. Полагая $x=\tau k, k=0,1,2, \mathrm{~K}$, где $\tau$ - период равномерной дискретизации функции, получаем ее дискретный аналог:

$$
\tilde{y}_{k}=a_{0} \exp (-\alpha \tau k)\left[\cos (\beta \tau k)+\frac{\alpha}{\beta} \sin (\beta \tau k)\right] .
$$

Применяя к обеим частям данного выражения $z$-преобразование, получаем: $Z\left\{\tilde{y}_{k}\right\}=a_{0} \frac{1-z^{-1} e^{-\alpha \tau}\left(\cos \beta \tau-\alpha \beta^{-1} \sin \beta \tau\right)}{1-\lambda_{1} z^{-1}+\lambda_{2} z^{-2}}, \quad$ где $\quad \lambda_{1}=2 e^{-\alpha \tau} \cos \beta \tau, \quad \lambda_{2}=-e^{-2 \alpha \tau} . \quad$ Отсюда $Z\left\{\tilde{y}_{k}\right\}-z^{-1} \lambda_{1} Z\left\{\tilde{y}_{k}\right\}-z^{-2} \lambda_{2} Z\left\{\tilde{y}_{k}\right\}=a_{0}-z^{-1} a_{0} e^{-\alpha \tau}\left(\cos \beta \tau-\alpha \beta^{-1} \sin \beta \tau\right)$. Возвращаясь в пространство оригиналов, используя первую теорему смещения: $z^{-r} Z\left\{\tilde{y}_{k}\right\}=\tilde{y}_{k-r}, r=0,1,2$, при условии, что $\tilde{y}_{k-r}=0$ при $k-r<0$, получаем разностное уравнение вида

$$
\tilde{y}_{k}-\lambda_{1} \tilde{y}_{k-1}-\lambda_{2} \tilde{y}_{k-2}=\delta_{k} a_{0}-\delta_{k-1} a_{0} e^{-\alpha \tau}\left(\cos \beta \tau-\alpha \beta^{-1} \sin \beta \tau\right), \quad k=0,1,2, \mathrm{~K},
$$

где $\delta_{k}=\left\{\begin{array}{l}1, \text { при } k=0 ; \\ 0, \text { при } k \neq 0\end{array} \quad\right.$ - символ Кронекера.

При $k=0$ и $k=1$ с учетом $\lambda_{4}=a_{0} \alpha \beta^{-1} e^{-\alpha \tau} \sin \beta \tau$ отсюда соответственно получаем

$$
\tilde{y}_{0}=a_{0}=\lambda_{3}, \quad \tilde{y}_{1}=a_{0} e^{-\alpha \tau} \cos \beta \tau+a_{0} \alpha \beta^{-1} e^{-\alpha \tau} \sin \beta \tau=\frac{\lambda_{1}}{2} \tilde{y}_{0}+\lambda_{4} .
$$

При $k \geq 2$ приходим к линейно параметрической дискретной модели в форме разностного уравнения второго порядка

$$
\tilde{y}_{k}=\lambda_{1} \tilde{y}_{k-1}+\lambda_{2} \tilde{y}_{k-2}=0, \quad k=2,3, \mathrm{~K},
$$

которое рекуррентно описывает временную последовательность дискретных значений корреляционной функции (2).

Коэффициенты в модели (4) связаны с параметрами корреляционной функции соотношениями:

$$
\lambda_{1}=2 e^{-\alpha \tau} \cos \beta \tau, \quad \lambda_{2}=-e^{-2 \alpha \tau}, \quad \lambda_{3}=a_{0}, \quad \lambda_{4}=a_{0} \alpha \beta^{-1} e^{-\alpha \tau} \sin \beta \tau .
$$

При обработке эксперимента формируется выборка результатов наблюдений $y_{k}$, $k=\overline{0, N-1}$, где $N$ - объем выборки. Результаты наблюдений $y_{k}$ содержат аддитивную случайную помеху $\varepsilon_{k}: y_{k}=\tilde{y}_{k}+\varepsilon_{k}$, где $\tilde{y}_{k}$ - дискретные значения, соответствующие теоретической корреляционной функции, и, следовательно, соотношениям (1) и (2). Тогда ЛПДМ в форме стохастического разностного уравнения можно представить в виде 


$$
\left\{\begin{array}{l}
y_{0}=\lambda_{3}+\varepsilon_{0}, \\
y_{1}=\lambda_{1} \frac{y_{0}}{2}+\lambda_{4}-\frac{\lambda_{1}}{2} \varepsilon_{0}+\varepsilon_{1}, \\
y_{k}=\lambda_{1} y_{k-1}+\lambda_{2} y_{k-2}+\eta_{k}, \\
\eta_{k}=-\lambda_{2} \varepsilon_{k-2}-\lambda_{1} \varepsilon_{k-1}+\varepsilon_{k}, \quad k=\overline{2, N-1}
\end{array}\right.
$$

В матричной форме ЛПДМ, описывающая корреляционную функцию при аддитивной случайной помехе в отсчетах наблюдений, имеет вид

$$
\left\{\begin{array}{l}
b=F \lambda+\eta \\
\eta=P \varepsilon
\end{array}\right.
$$

или в развернутой форме обобщенной регрессионной модели -

$$
\begin{aligned}
& b_{i}=\lambda_{1} f_{i 1}+\lambda_{2} f_{i 2}+\lambda_{3} f_{i 3}+\lambda_{4} f_{i 4}+\eta_{i}, \\
& \eta_{i}=p_{i 1} \varepsilon_{0}+p_{i 2} \varepsilon_{1}+p_{i 3} \varepsilon_{2}+\mathrm{K}+p_{i, N-1} \varepsilon_{N-2}+p_{i N} \varepsilon_{N-1}, \quad i=\overline{1, N} .
\end{aligned}
$$

Здесь $\lambda=\left(\lambda_{1}, \lambda_{2}, \lambda_{3}, \lambda_{4}\right)^{T}$ - вектор неизвестных коэффициентов ЛПДМ; $\varepsilon=\left(\varepsilon_{0}, \varepsilon_{1}, \mathrm{~K}, \varepsilon_{N-1}\right)^{T}-$ $N$ - мерный вектор случайной помехи в результатах наблюдений; $\eta=\left(\eta_{1}, \eta_{2}, \mathrm{~K}, \eta_{N}\right)^{T}-N$ мерный вектор эквивалентного возмущения в стохастическом разностном уравнении; $b=\left(y_{0}, y_{1}, y_{2}, \mathrm{~K}, y_{k-1}, \mathrm{~K}, y_{N-1}\right)^{T}-N$ - мерный вектор правой части; $F$ - матрица регрессоров размера $N \times 4$, столбцы которой описываются формулами:

$f_{i 1}=\left(0, \frac{y_{0}}{2}, y_{1}, y_{2}, \mathrm{~K}, y_{k-2}, \mathrm{~K}, y_{N-2}\right)^{T}, f_{i 2}=\left(0,0, y_{0}, y_{1}, y_{2}, \mathrm{~K}, y_{k-3}, \mathrm{~K}, y_{N-3}\right)^{T}, f_{i 3}=(1,0, \mathrm{~K}, 0)^{T}$, $f_{i 4}=(0,1,0, \mathrm{~K}, 0)^{T} ; P$ - матрица размера $N \times N$ в стохастическом уравнении эквивалентного возмущения- нижняя треугольная, ленточная, трех диагональная. Первый столбец матрицы $P$ имеет вид: $p_{i 1}=\left(1,-\frac{\lambda_{1}}{2},-\lambda_{2}, 0,0, \mathrm{~K}, 0\right)^{T}$. Остальные столбцы матрицы $P, j=\overline{2, N}$, описываются формулами:

$$
p_{i j}=\left\{\begin{array}{cll}
0, & 1 \leq i<j, & j=\overline{2, N}, \\
1, & i=j, & j=\overline{2, N} \\
-\lambda_{1}, & i=j+1, & j=2, N-1 \\
-\lambda_{2}, & i=j+2, & j=\overline{2, N-2} \\
0, & j+3 \leq i \leq N, & j=2, N-3
\end{array}\right.
$$

Построенная линейно параметрическая дискретная модель в форме системы из двух стохастических разностных уравнения (7) или (8) и (9) лежит в основе предлагаемого метода определения параметров корреляционной функции распределения случайного поля неупругой реологической деформации.

Определение параметров корреляционной функции основе стохастического разностного уравнения. Исходными данными, на основе которых определяются параметры корреляционной функции, служат результаты вычислений, полученные в процессе физического эксперимента. Проводя анализ экспериментальных данных, например [1], представленных для математической обработки, следует отметить, во-первых, небольшие объемы выборок: $N \leq 20$, а, во-вторых, то, что эти выборки охватывают не более одного периода корреляционной функции: $T=\frac{2 \pi}{\beta}$. Очевидно, что при этом период дискретизации $\tau$, используемый при формировании экспериментальных данных, сравнительно мал (в относительных к периоду корреляционной функции единицах). Это отрицательно влияет на устойчивость вычислений среднеквадратичных оценок коэффициентов ЛПДМ и должно быть учтено при разработке численного метода определения параметров корреляционной функции.

Полученные в ходе эксперимента результаты вычислений $y_{k}$ содержат аддитивную случайную помеху $\varepsilon_{k}$. Будем предполагать, что случайные возмущения $\varepsilon_{k}$ имеют нулевое математическое ожидание, дисперсию $\sigma_{\varepsilon}^{2}$, не коррелированны между собой (матрица дисперсий- 
ковариаций: $V[\varepsilon]=\sigma_{\varepsilon}^{2} E$, где $E$ - единичная матрица) и подчиняются нормальному закону распределения: $\varepsilon_{k} \in N\left(0, \sigma_{\varepsilon}^{2}\right)$.

Алгоритм определения параметров корреляционной функции на основе среднеквадратичных оценок коэффициентов стохастического разностного уравнения включает следующие основные этапы.

1. Формирование на основе ЛПДМ системы линейных алгебраических уравнений. На этом этапе по приведенным выше формулам, описывающим элементы стохастического разностного уравнения (8), вычисляются элементы матрицы $F=\left(f_{i j}\right), j=\overline{1,4}$ и правой части $b=\left(b_{i}\right)$, $i=\overline{1, N}$, переопределенной системы линейных алгебраических уравнений относительно коэффициентов $\lambda_{j}, j=\overline{1,4}$.

2. Вычисление среднеквадратичных оценок коэффициентов стохастического разностного уравнения. Данный этап является определяющим в процессе формирования погрешности оценок параметров корреляционной функции. От того, насколько обоснованно и корректно будет выбран метод среднеквадратичного оценивания, существенно зависит точность вычисления коэффициентов ЛПДМ. Разработка и исследование эффективного численного итерационного метода среднеквадратичного оценивания коэффициентов стохастического разностного уравнения (8) является основной задачей, решаемой в рамках данной работы.

3. Вычисление параметров корреляциионой функции распределения случайного поля неупругой реологической деформации. С учетом известных соотношений (5) по среднеквадратичным оценкам $\hat{\lambda_{i}}$ коэффициентов ЛПДМ вычисляются параметры корреляционной функции:

$$
\alpha=-\frac{1}{2 \tau} \ln \left(-\hat{\lambda_{2}}\right), \quad \beta=\frac{1}{\tau} \arccos \frac{\hat{\lambda_{1}}}{2 \sqrt{-\hat{\lambda}_{2}}}, \quad a_{0}=\hat{\lambda_{3}} .
$$

4. Оченка погрешности результатов вычислений. На заключительном этапе алгоритма определения параметров корреляционной функции оценивается погрешность полученных результатов. Так как в алгоритме вычислений применяются статистические методы обработки экспериментальных данных, то, очевидно, что погрешность носит случайный характер, и для ее описания можно использовать среднее квадратическое отклонение оценок параметров корреляционной функции. Построение доверительных интервалов для этих оценок позволит указать предельные (с заданной доверительной вероятностью) абсолютную и относительную погрешности.

Разработка и исследование итерационного метода среднеквадратичного оценивания коэффициентов стохастического разностного уравнения. Применение статистических методов при вычислении коэффициентов $\lambda_{j}$ линейно параметрической дискретной модели позволяет существенно повысить точность оценивания. Однако вычисление среднеквадратичных оценок $\hat{\lambda}_{j}$ по обобщенной регрессионной модели, которая описывается уравнением (8): $\sum_{i=1}^{N}\left[b_{i}-\sum_{j=1}^{4} \lambda_{j} f_{i, j+1}\right]^{2}=\sum_{i=1}^{N} \eta_{i}^{2} \Rightarrow \min$, приводит к значительному асимптотическому смещению в оценках из-за корреляции между отсчетами случайного эквивалентного возмущения $\eta_{i}$ и элементами матрицы $f_{i j}, i=\overline{1,2}$, [6]. Этот существенный недостаток устраняется за счет использования итерационной процедуры при вычислении среднеквадратичных оценок.

Из (10) очевидно, что всегда существует обратная матрица $P_{\lambda}^{-1}$. Тогда после преобразования обобщенной регрессионной модели (7) получаем $P_{\lambda}^{-1} b=P_{\lambda}^{-1} F \lambda+\varepsilon$. Вычисление среднеквадратичных оценок коэффициентов $\hat{\lambda}_{j}$ на основе минимизации функционала $J_{\lambda}=\left\|P_{\lambda}^{-1} b-P_{\lambda}^{-1} F \lambda\right\|^{2}=\|\varepsilon\|^{2} \Rightarrow \min$ позволяет устранить асимптотическое смещение, связанное с корреляцией между случайным возмущением и результатами наблюдений, и тем самым повысить точность вычисления параметров корреляционной функции. Так как элементы матрицы $P_{\lambda}^{-1}$ зависят от $\lambda_{1}$ и $\lambda_{2}$, то, в общем случае, минимизация функционала $J_{\lambda}$ приводит к решению системы нелинейных относительно $\lambda_{j}$ уравнений. Рассмотрим итерационную процедуру вычисления среднеквадратичных оценок коэффициентов $\lambda_{j}$. На первом этапе вычисляются 
первоначальные МНК - оценки $\lambda_{j}^{(0)}:\|b-F \lambda\|^{2}=\|\eta\|^{2} \Rightarrow \min$. Затем формируется приближение матрицы $P_{\lambda} \approx P_{\lambda}^{(1)}\left(\lambda_{1}^{(0)}, \lambda_{2}^{(0)}\right)$, преобразуется обобщенная регрессионная модель (7), и вычисляются новые, уточненные оценки $\lambda_{j}^{(1)}$ коэффициентов ЛПДМ. Эти оценки используются для вычисления второго приближения матрицы $P_{\lambda} \approx P_{\lambda}^{(2)}\left(\lambda_{1}^{(1)}, \lambda_{2}^{(1)}\right)$ и т.д. Процесс уточнений повторяется до тех пор, пока не будет выполнено условие останова, например: $\left\|\lambda^{(i)}-2 \lambda^{(i-1)}+\lambda^{(i-2)}\right\|<0,01\left\|\lambda^{(i)}\right\|$. Найденные на последней итерации оценки $\lambda^{(i)}$ принимаются за истинные значения коэффициентов ЛПДМ и по ним далее вычисляются параметры корреляционной функции распределения случайного поля неупругой реологической деформации.

Проведены численно-аналитические исследования сходимости описанной итерационной процедуры. Численный эксперимент был организован следующим образом. Генерировалась выборка $\tilde{y}_{k}$ дискретных значений функции (2) с параметрами $a_{0}=1, \alpha=0,5, \beta=2$. Период дискретизации $\tau=0,15$ и объем выборки $N=20$ при формировании тестового сигнала выбирались в соответствии с режимом проведения физического эксперимента, в частности: $\frac{\tau}{T}=0,048$, где $T=\frac{2 \pi}{\beta}-$ период корреляционной функции. В отсчеты $\tilde{y}_{k}$, соответствующие точным значениям функции, добавлялась аддитивная помеха $\varepsilon_{k}$, значения которой обеспечивали заданную величину мощности помехи $3 \%, 5 \%$ и $10 \%$ в относительных единицах к мощности сигнала: $\frac{\|\varepsilon\|}{\|\tilde{y}\|} 100 \%$. Результаты вычислений параметров корреляционной функции усреднялись по 1000 реализациям.

На рис. 1 и рис. 2 представлены зависимости относительной погрешности вычисления параметра $\alpha$, характеризующего затухание корреляционной функции, и параметра $\beta$ - частоты корреляционной функции, от числа итераций $n$ для различных значений мощности случайной помехи в результатах наблюдений. Кривые 1, 2 и 3 построены по результатам вычислений при мощности случайной помехи $3 \%, 5 \%$ и $10 \%$. Точки на графиках при $n=0$ соответствуют результатам среднеквадратичного оценивания без предварительного преобразования обобщенной регрессионной модели (7).

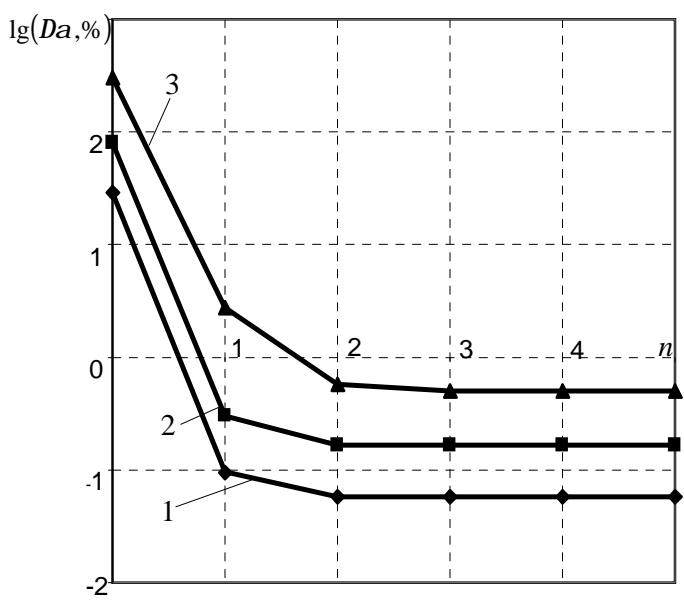

Р и с. 1 Зависимость относительной погрешности вычисления параметра $\alpha$ от числа итераций (в логарифмическом массштабе)

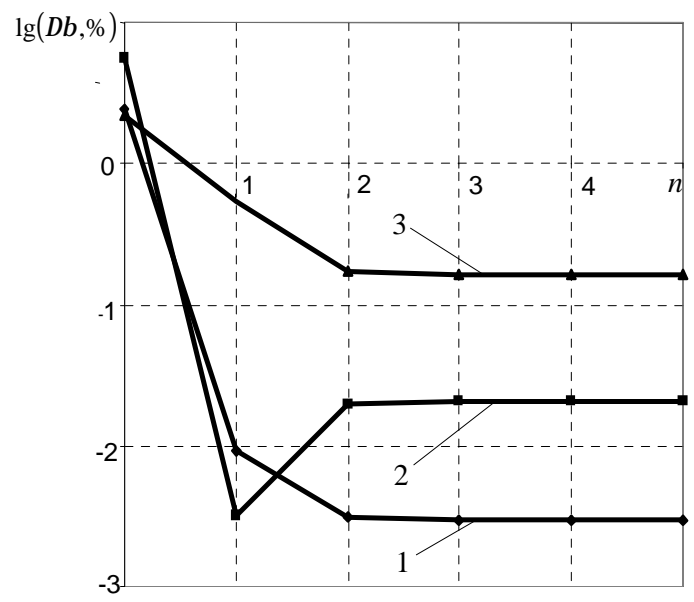

Р и с. 2 Зависимость относительной погрешности вычисления параметра $\beta$ от числа итераций (в логарифмическом массштабе) 
По результатам исследований можно сделать выводы, что, во-первых, применение итерационного метода среднеквадратичного оценивания позволяет существенно, в сотни раз, уменьшить погрешность вычисления параметров корреляционной функции. Во-вторых, результаты вычислений практически не изменяются после второй итерации, что свидетельствует о хорошей сходимости метода. При этом точность оценивания параметра $\beta$ - частоты корреляционной функции - на два порядка выше, чем параметра $\alpha$.

Проведены исследования эффективности предложенного итерационного метода вычисления среднеквадратичных оценок. Целью таких исследований являлся сравнительный анализ погрешности вычисления параметров корреляционной функции двумя различными способами: на основе среднеквадратичных оценок коэффициентов модели (8) и на основе итерационного численного метода. Для этого, как и в предыдущем случае, формировалась выборка дискретных значений тестового сигнала с параметрами $a_{0}=1, \alpha=0,5, \beta=2, \tau=0,15, N=20$, что соответствует рабочей области проведения физического эксперимента по изучению неупругой реологической деформации. В отсчеты тестового сигнала добавлялась случайная помеха, мощность которой изменялась в диапазоне от 0 до $20 \%$.

В качестве критериев эффективности в сравнительном анализе методов среднеквадратичного оценивания использовались:

- смещение оценок $\hat{\alpha}$ и $\hat{\beta}$ относительно истинных значений (в \%): $\Delta \alpha=\frac{|M[\hat{\alpha}]-\alpha|}{|\alpha|} 100 \%$;

- дисперсии оценок параметров корреляционной функции: $D[\hat{\alpha}]=M\left[(\hat{\alpha}-M[\hat{\alpha}])^{2}\right]$;

- обобщенная ошибка вычисления коэффициентов разностного уравнения - второй момент оценки относительно истинного значения коэффициента: $M\left[(\hat{\lambda}-\lambda)^{2}\right]=D[\hat{\lambda}]+(M[\hat{\lambda}]-\lambda)^{2}$.

Здесь $M[\cdot]$ - оператор математического ожидания, статистическая оценка которого в каждом случае находилась посредством усреднения $M=1000$ реализаций для каждой точки эксперимента.

На рис. 3, 4 представлены зависимости смещения оценок параметров корреляционной функции (в относительных единицах) от мощности случайной помехи. Так как результаты вычислений различаются в сотни, тысячи и более раз, то для оси ординат используется логарифмический масштаб. Точки 1 соответствуют методу наименьших квадратов, примененному к модели (8) без ее преобразования, а точки 2 - итерационному методу вычислений среднеквадратичных оценок коэффициентов ЛПДМ. Очевидно, что точность вычисления при использовании итерационного метода среднеквадратичного оценивания на несколько порядков выше по сравнению с методом наименьших квадратов, примененному непосредственно к модели (8). Это достигается за счет устранения большого смещения в МНК - оценках, обусловленного корреляцией между случайной эквивалентной помехой $\eta$ и элементами матрицы $F$.

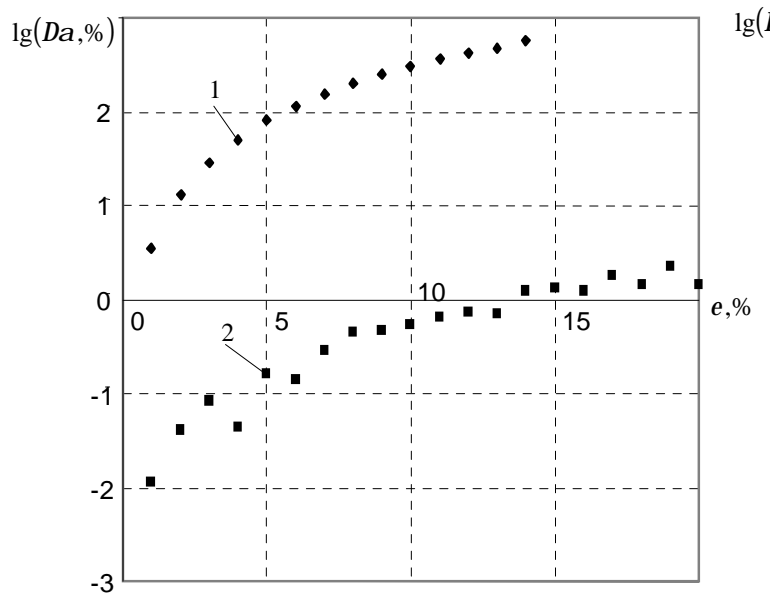

Р и с. 3. Зависимость смещения оценки параметра $\alpha$ от величины случайной помехи в результатах наблюдений (в логарифмическом масштабе)

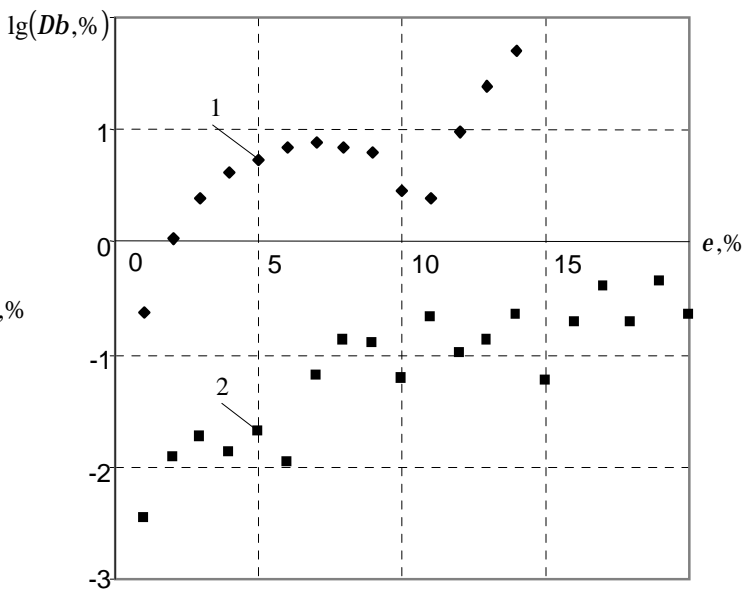

Р и с. 4. Зависимость смещения оценки параметра $\beta$ от величины случайной помехи в результатах наблюдений (в логарифмическом масштабе) 
Несмещенность оценок на практике не всегда гарантирует высокую точность. Большое значение имеет и дисперсия оценок, которая зависит от выбора параметров обработки экспериментальных данных. Проведены исследования зависимости дисперсий оценок коэффициентов ЛПДМ и параметров корреляционной функции от мощности случайной помехи в результатах эксперимента. Выборочные дисперсии оценок коэффициентов ЛПДМ $s^{2}\left[\hat{\lambda}_{1}^{\wedge}\right]$ и $s^{2}\left[\hat{\lambda}_{2}\right]$ вычислялись по $M=1000$ реализациям для каждой точки эксперимента: $s^{2}\left[\hat{\lambda_{j}}\right]=\frac{\sum_{p=1}^{M}\left(\hat{\lambda}_{j p}-\bar{\lambda}_{j}\right)^{2}}{M-1}$, $\bar{\lambda}_{j}=\frac{\sum_{p=1}^{M} \hat{\lambda}_{j p}}{M}, j=1,2$. Оценки дисперсий для параметров корреляционной функции определялись по формулам:

$$
\begin{gathered}
s^{2}[\hat{\alpha}] \approx\left|\frac{\partial \alpha}{\partial \hat{\lambda}_{2}}\right|^{2} s^{2}\left[\hat{\lambda}_{2}\right] \\
s^{2}[\hat{\beta}] \approx\left|\frac{\partial \beta}{\partial \hat{\lambda}_{1}}\right|^{2} s^{2}\left[\hat{\lambda_{1}}\right]+2 \frac{\partial \beta}{\partial \hat{\lambda}_{1}} \frac{\partial \beta}{\partial \hat{\lambda}_{2}} \operatorname{cov}\left[\hat{\lambda_{1}}, \hat{\lambda_{2}}\right]+\left|\frac{\partial \beta}{\partial \hat{\lambda}_{2}}\right|^{2} s^{2}\left[\hat{\lambda_{2}}\right],
\end{gathered}
$$

в которых в соответствии с (11) $\frac{\partial \alpha}{\partial \hat{\lambda}_{2}}=-\frac{1}{2 \tau \hat{\lambda_{2}}}, \frac{\partial \beta}{\partial \hat{\lambda}_{1}}=-\frac{1}{\tau \sqrt{-4 \hat{\lambda_{2}}-\hat{\lambda}_{1}^{2}}}$ и $\frac{\partial \beta}{\partial \hat{\lambda}_{2}^{\hat{n}}}=\frac{\lambda_{1}}{2 \tau \lambda_{2} \sqrt{-4 \hat{\lambda_{2}}-\hat{\lambda}_{1}^{2}}}$, а выборочная ковариация между оценками $\hat{\lambda}_{1}$ и $\hat{\lambda}_{2}$ вычислялась по формуле $\operatorname{cov}\left[\hat{\lambda}_{1}, \hat{\lambda}_{2}\right]=\frac{\sum_{p=1}^{M}\left(\hat{\lambda}_{1 p}-\bar{\lambda}_{1}\right)\left(\hat{\lambda}_{2 p}-\bar{\lambda}_{2}\right)}{M-1}$.

На рис. 5, 6 представлены зависимости (в логарифмическом масштабе) среднего квадратического отклонения оценок параметров корреляционной функции от величины случайной помехи в результатах наблюдений. Очевидно, что применение итерационного метода (точки 2) позволило на два порядка уменьшить дисперсии по сравнению со среднеквадратичным оцениванием по модели (8) без ее преобразования (точки 1).

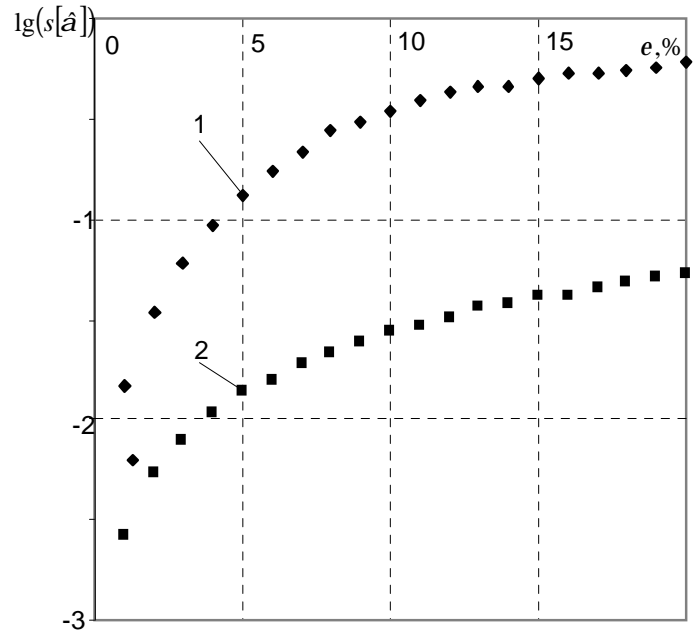

Р и с. 5 Зависимость среднего квадратического отклонения оценки параметра $\alpha$ от величины случайной помехи в результатах наблюдений (в логарифмическом масштабе)

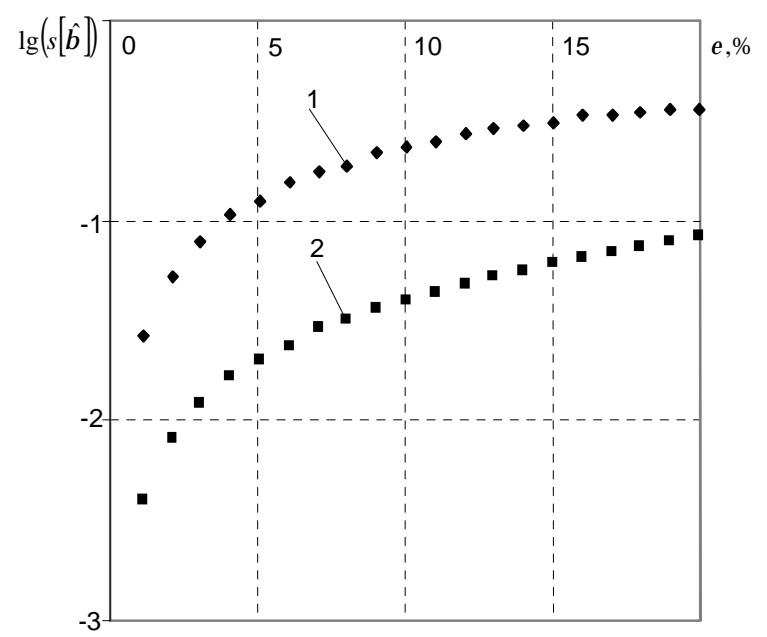

Р и с. 6 Зависимость среднего квадратического отклонения оценки параметра $\beta$ от величины случайной помехи в результатах наблюдений (в логарифмическом масштабе)

В процессе формирования результирующей ошибки заметную роль играет погрешность среднеквадратичного оценивания коэффициентов $\lambda_{j}$ ЛПДМ. При фиксированной выборке результатов эксперимента эта погрешность зависит от смещения и дисперсии оценки. Причем 
требование несмещенности на практике не всегда оправдано, так как оценка с небольшим смещением и малой дисперсией может оказаться предпочтительнее несмещенной оценки, но с большей дисперсией. Поэтому целесообразно использовать обобщенный показатель ошибки второй момент оценки относительно истинного значения параметра. На рис. 7, 8 представлены зависимости (в логарифмическом масштабе) обобщенной ошибки вычисления коэффициентов $\lambda_{1}$ и $\lambda_{2}$ от величины случайной помехи в результатах наблюдений. Точки 1 соответствуют среднеквадратичным оценкам, полученным на основе модели (8) без ее преобразования, а точки 2 - итерационному методу. Как и в предыдущих случаях, применение итерационного метода среднеквадратичного оценивания существенно, на несколько порядков, позволяет повысить точность вычислений.

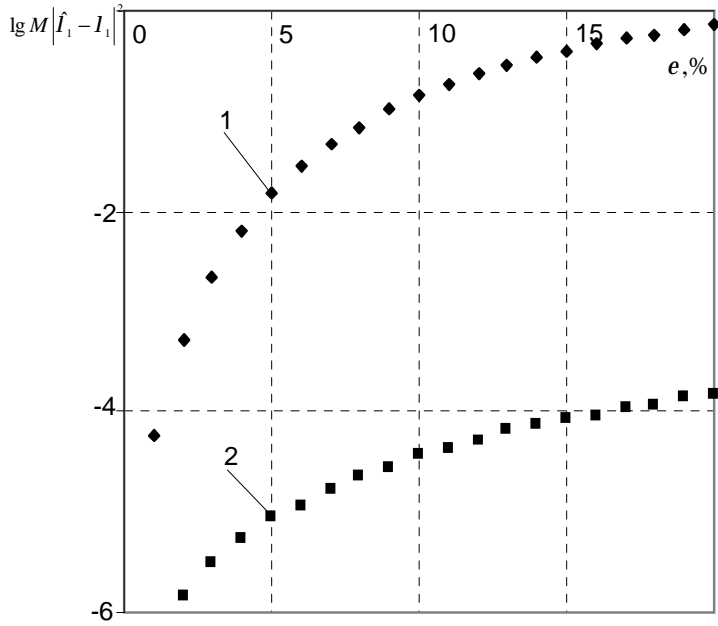

Р и с. 7 Зависимость обобщенной ошибки вычисления коэффициента $\lambda_{1}$ от величины случайной помехи в результатах наблюдений (в логарифмическом масштабе)

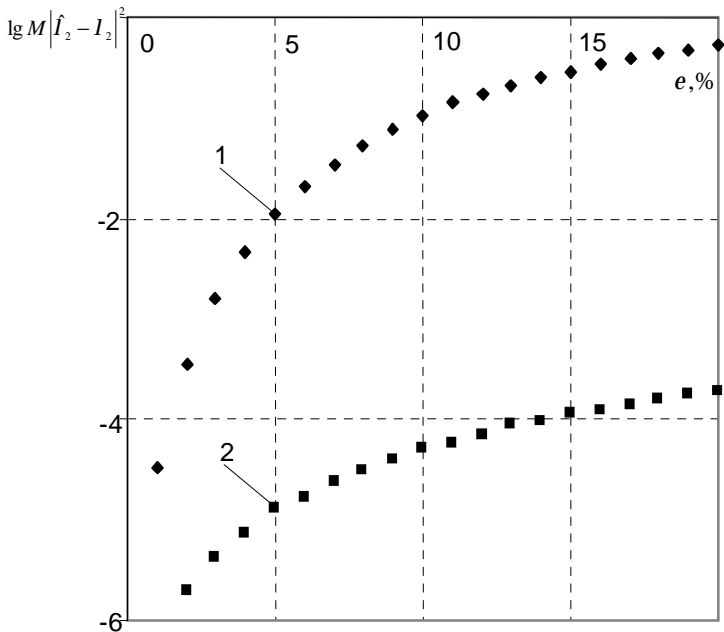

Р и с. 8 Зависимость обобщенной ошибки вычисления коэффициента $\lambda_{2}$ от величины случайной помехи в результатах наблюдений (в логарифмическом масштабе)

Таким образом, проведенные численно-аналитические исследования помехозащищенности итерационного численного метода, в основе которого лежит линейно параметрическая дискретная модель в форме стохастического разностного уравнения, показали его высокую эффективность при вычислении параметров корреляционной функции распределения случайного поля неупругой реологической деформации.

Оценка погрешности вычисления параметров корреляционной функции в процессе обработки результатов эксперимента. Основным источником погрешности вычисления параметров корреляционной функции является случайная помеха в результатах эксперимента, поэтому для оценки погрешности целесообразно использовать статистические методы [7]. Процедуру вычисления результирующей погрешности можно разбить на следующие основные этапы. Вначале на основе статистической обработки результатов оценивания коэффициентов обобщенной регрессионной модели в форме стохастических разностных уравнений (8) и (9) находятся оценки дисперсий $s^{2}\left\lfloor\hat{\lambda_{j}}\right\rfloor$ для коэффициентов $\lambda_{j}, j=\overline{1,3}$. Затем с учетом формул (12) и (13) вычисляются оценки дисперсий $s^{2}[\hat{\alpha}], s^{2}[\hat{\beta}]$ и $s^{2}\left[\hat{a}_{0}\right]$ параметров корреляционной функции. На заключительном этапе вычисляются доверительные границы случайной погрешности, которые могут быть интерпретированы как предельные абсолютные погрешности $\Delta \alpha, \Delta \beta$ и $\Delta a_{0}$ (с заданной доверительной вероятностью) для оценок параметров корреляционной функции. Полученные результаты позволят также найти и предельные (с заданной доверительной вероятностью) относительные погрешности вычисления $\delta \alpha=\frac{\Delta \alpha}{|\hat{\alpha}|}, \delta \beta=\frac{\Delta \beta}{|\hat{\beta}|}$ и $\delta a_{0}=\frac{\Delta a_{0}}{\left|\hat{a}_{0}\right|}$ параметров корреляционной функции. 
При использовании итерационного метода вычисления среднеквадратичных оценок коэффициентов ЛПДМ минимизируется функционал $J_{\lambda}=\left\|P_{\lambda}^{-1} b-P_{\lambda}^{-1} F \lambda\right\|^{2}=\|\varepsilon\|^{2} \Rightarrow \min$. Это приводит к оценкам $\hat{\lambda}=\left(F^{T} \Omega_{\lambda}^{-1} F\right)^{-1} F^{T} \Omega_{\lambda}^{-1} b$, где матрица $\Omega_{\lambda}=P_{\lambda} P_{\lambda}^{T}$. Отсюда можно получить $\hat{\lambda}=\lambda+\left(F^{T} \Omega_{\lambda}^{-1} F\right)^{-1} F^{T}\left(P_{\lambda}^{-1}\right)^{T} \varepsilon . \quad$ Тогда $\quad M[\hat{\lambda}]=\lambda+M\left[\left(F^{T} \Omega_{\lambda}^{-1} F\right)^{-1} F^{T}\left(P_{\lambda}^{-1}\right)^{T} \varepsilon\right]=\lambda+\Delta \hat{\lambda}, \quad$ где $\Delta \hat{\lambda}=M\left[\left(F^{T} \Omega_{\lambda}^{-1} F\right)^{-1} F^{T}\left(P_{\lambda}^{-1}\right)^{T} \varepsilon\right]-$ смещение, обусловленное корреляцией между случайным возмущением $\varepsilon$ и элементами матрицы $F$. Так как при данном алгоритме оценивания смещение мало и практически равно нулю (что подтверждается результатами не только численных, но и аналитических исследований), то в первом приближении $M[\hat{\lambda}]=\lambda$.

Рассмотрим ковариационную матрицу оценок $\hat{\lambda}: V[\hat{\lambda}]=M\left[(\hat{\lambda}-M[\hat{\lambda}])(\hat{\lambda}-M[\hat{\lambda}])^{T}\right]$. Используя линейную аппроксимацию вектора $\hat{\lambda}-\lambda=\left(F^{T} \Omega_{\lambda}^{-1} F\right)^{-1} F^{T}\left(P_{\lambda}^{-1}\right)^{T} \varepsilon \approx\left(\tilde{F}^{T} \Omega_{\lambda}^{-1} \tilde{F}\right)^{-1} \tilde{F}^{T}\left(P_{\lambda}^{-1}\right)^{T} \varepsilon$, где $\tilde{F}$ - неслучайная матрица регрессоров, элементы которой описываются теми же формулами, что и для матрицы $F$ с учетом замены $y_{k}=\tilde{y}_{k}+\varepsilon_{k}$ на $\tilde{y}_{k}$, получаем: $V[\hat{\lambda}]=M\left[(\hat{\lambda}-\lambda)(\hat{\lambda}-\lambda)^{T}\right]=M\left[\left(\tilde{F}^{T} \Omega_{\lambda}^{-1} \tilde{F}\right)^{-1} \tilde{F}^{T}\left(P_{\lambda}^{-1}\right)^{T} \varepsilon \varepsilon^{T} P_{\lambda}^{-1} \tilde{F}\left(\tilde{F}^{T} \Omega_{\lambda}^{-1} \tilde{F}\right)^{-1}\right]=\left(\tilde{F}^{T} \Omega_{\lambda}^{-1} \tilde{F}\right)^{-1} M\left[\varepsilon \varepsilon^{T}\right]=$ $=\left(\tilde{F}^{T} \Omega_{\lambda}^{-1} \tilde{F}\right)^{-1} \sigma_{\varepsilon}^{2} \approx\left(F^{T} \Omega_{\lambda}^{-1} F\right)^{-1} \sigma_{\varepsilon}^{2}$. Обозначив элементы матрицы $C=\left(F^{T} \Omega_{\lambda}^{-1} F\right)^{-1}$ через $c_{i j}$, $i=\overline{1,4}, j=\overline{1,4}$, дисперсии и ковариации коэффициентов $\hat{\lambda_{i}}$ можно оценить по формулам $s^{2}\left[\hat{\lambda}_{i}\right]=c_{i i} s^{2}, \operatorname{cov}\left[\hat{\lambda}_{i}, \hat{\lambda_{j}}\right]=c_{i j} s^{2}$, где $s^{2}$ - оценка дисперсии $\sigma_{\varepsilon}^{2}$ случайного возмущения в результатах наблюдений. При обработке экспериментальных данных в соответствии с данной методикой дисперсию случайного возмущения можно оценить по выражению $s^{2}=\frac{\sum_{k=1}^{N}\left(e_{k}-\bar{e}\right)^{2}}{N-4}$, $\bar{e}=\frac{1}{N} \sum_{k=1}^{N} e_{k}$, где $e_{k}-$ остатки, которые находятся через оценки вектора коэффициентов ЛПДМ по формуле $e=P_{\lambda}^{-1} b-P_{\lambda}^{-1} F \hat{\lambda}$.

По известным оценкам дисперсий и ковариаций коэффициентов ЛПДМ можно найти оценки средних квадратических отклонений параметров корреляционной функции. Из формул (12) и (13) следует $s[\hat{\alpha}]=\frac{1}{2 \tau\left|\hat{\lambda}_{2}\right|} s\left[\hat{\lambda}_{2}\right], \quad s[\hat{\beta}]=\frac{1}{2 \tau\left|\hat{\lambda_{2}}\right| \sqrt{-4 \hat{\lambda}_{2}-\hat{\lambda}_{1}^{2}}} \sqrt{4 \hat{\lambda}_{2}^{2} s^{2}\left[\hat{\lambda}_{1}\right]-4 \hat{\lambda}_{1} \hat{\lambda}_{2} \operatorname{cov}\left[\hat{\lambda}_{1}, \hat{\lambda}_{2}\right]+\hat{\lambda}_{1}^{2} s^{2}\left[\hat{\lambda}_{2}\right]}$ и $s\left[a_{0}\right]=s\left[\hat{\lambda_{3}}\right]$.

В качестве оценки предельной абсолютной погрешности вычисления параметров корреляционной функции (с доверительной вероятностью $p$ ) можно использовать границу доверительного интервала, которая для $\alpha$, например, вычисляется по формуле: $\Delta \hat{\alpha}=t_{T} s[\hat{\alpha}]$ [6]. Здесь величина $t_{T}=t(\gamma, v)$ берется из таблицы распределения Стьюдента при числе степеней свободы $v=N-4$ и уровне значимости $\gamma=1-p$. При доверительной вероятности $p=0,99$ и объеме выборки $N \geq 15$ принимаем $t_{T}=2,7$. Границы доверительных интервалов для остальных параметров вычисляются по аналогичным формулам.

Проведены численно-аналитические исследования, подтверждающие достоверность полученных формул для оценки предельных абсолютной и относительной погрешностей вычисления параметров корреляционной функции при обработке экспериментальных данных. На рис. 9 - 10 кривые $1-3$ описывают границы для предельной относительной погрешности вычисления параметров $\alpha$ и $\beta$, построенные по десяти реализациям для каждой точки эксперимента. Кривые 1 и 3 соответствуют максимальному и минимальному значениям предельной относительной погрешности, а кривая 2 - значению, усредненному по десяти реализациям. 
Точки 4 на этих графиках отображают результаты вычислений действительной относительной погрешности: по 10 значений в каждой точке численного эксперимента. Очевидно, что погрешность оценок параметров корреляционной функции практически полностью укладывается в границы для предельной относительной погрешности, вычисленные в соответствии с предложенной методикой по представленным выше формулам.
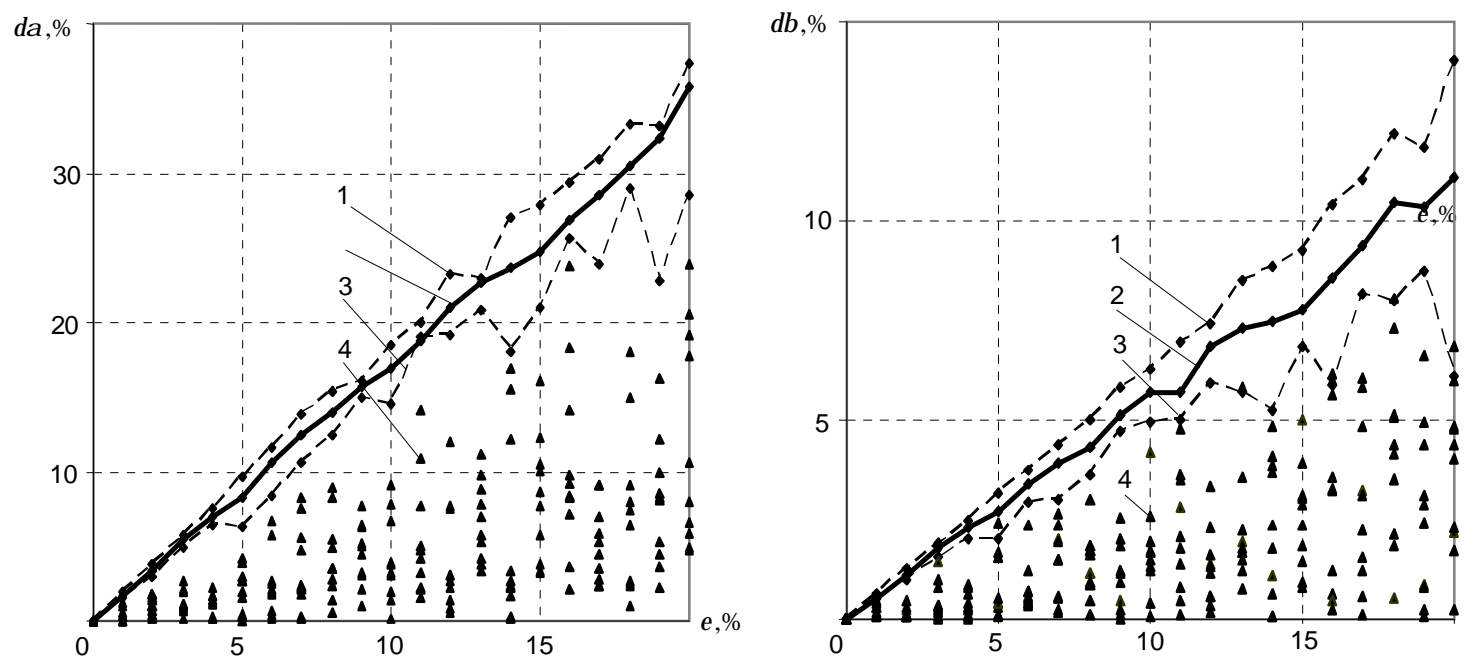

Р и с. 9 Зависимости оценок предельной относи- Р и с. 10 Зависимости оценок предельной относительной погрешности (кривые 1, 2 и 3) и относи- тельной погрешности (кривые 1, 2 и 3) и относительных погрешностей (точки 2) вычисления па- тельных погрешностей (точки 2) вычисления параметра $\alpha$ от величины случайной помехи раметра $\beta$ от величины случайной помехи

Применение итерационного метода среднеквадратичного оценивания параметров корреляционной функции при исследовании ползучести материалов. Разработанный метод оценивания параметров корреляционной функции на основе итерационной процедуры среднеквадратичного оценивания коэффициентов стохастического разностного уравнения был применен в научно-техническом эксперименте при исследовании ползучести материалов. Результаты измерений, полученные в ходе физического эксперимента и лежащие в основе оценивания параметров корреляционной функции распределения случайного поля неупругой реологической деформации, представлены в таблице.

Результаты эксперимента для построения корреляционной функции распределения случайного поля неупругой реологической деформации

\begin{tabular}{|c|c|c|c|c|c|c|c|c|c|c|}
\hline$x($ мM $)$ & 0 & 2 & 4 & 6 & 8 & 10 & 12 & 14 & 16 & 18 \\
\hline$K(x) 10^{6}$ & 195 & 88 & 51 & -6 & 31 & 28 & -11 & -99 & -129 & -115 \\
\hline$x($ Mм $)$ & 20 & 22 & 24 & 26 & 28 & 30 & 32 & 34 & 36 & \\
\hline$K(x) 10^{6}$ & -98 & -52 & -102 & -15 & -3 & 44 & 10 & 93 & 99 \\
\hline
\end{tabular}

Применение итерационного метода среднеквадратичного оценивания к выборке результатов эксперимента объема $N=19$ и с шагом дискретизации $\tau=2$ мм позволило получить следующие оценки параметров корреляционной функции: $\alpha=0,014, \beta=0,16$ и $a_{0}=135 \cdot 10^{-6}$. Предельные относительные погрешности вычисления этих параметров соответственно составляют 191\%, 19\% и 46\%. На рис. 11 точки 1 соответствуют результатам эксперимента, представленным в табл. 1. Кривая 2 является графиком корреляционной функции, восстановленной по экспериментальным данным. Для сравнения на этом же рисунке представлена кривая 3 , описывающая корреляционную функцию, параметры которой найдены методом наименьших квадратов, примененным непосредственно к нелинейной функции (2): $\sum_{k=1}^{N}\left(y_{k}-\tilde{y}_{k}\right)^{2}=\sum_{k=1}^{N} \varepsilon_{k}^{2} \Rightarrow \min$. При этом решение нелинейной системы нормальных уравнений в МНК находилось методом Ньютона с помощью пакета поиска решений из приложения Microsoft Excel. Однако решить 
задачу таким способом оказалось возможным только на основе первоначального приближения, полученного опять же итерационным численным методом среднеквадратичного оценивания коэффициентов ЛПДМ. Пусть $H_{0}$ - нулевая гипотеза: функции, представленные на рис. 11 кривыми 2 и одинаково хорошо аппроксимируют экспериментальные данные 1 . Для проверки этой гипотезы найдено отношение остаточных дисперсий $F=1,30$, которое имеет распределение Фишера. При уровне значимости $\gamma=0,05$ и соответствующих числах степеней свободы $F_{\kappa p}=2,33>F$. Следовательно, можно считать, что по отношению к экспериментальным данным различие между этими двумя функциями мало и обусловлено только случайной помехой в результатах наблюдений.

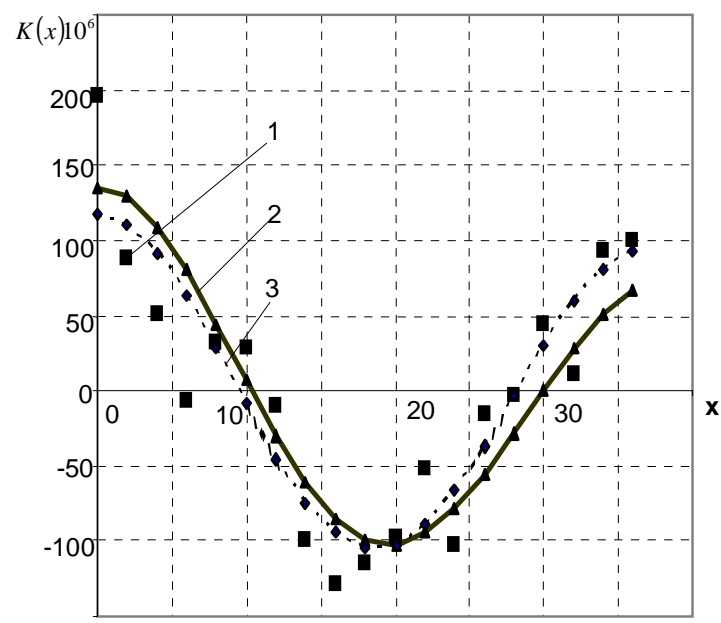

Р и с. 11 Восстановленная по экспериментальным данным корреляционная функция распределения случайного поля реологической деформации при исследовании ползучести материала

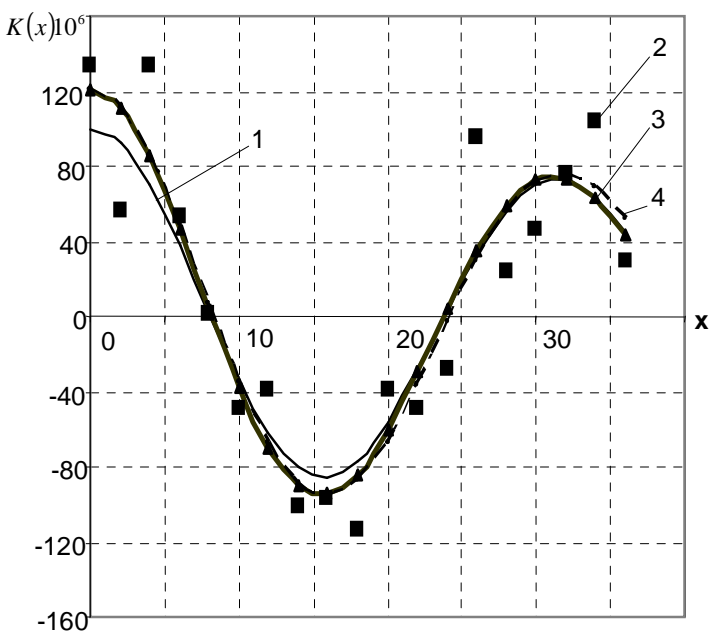

Р и с. 12 Моделирование корреляционной функции распределения случайного поля реологической деформации при исследовании ползучести материала

Проведено численно-аналитическое моделирование результатов данного эксперимента с целью анализа статистических характеристик погрешности оценок параметров корреляционной функции. При моделировании генерировался тестовый сигнал (2) с параметрами близкими к параметрам восстановленной по экспериментальным данным корреляционной функции: $\alpha=0,01, \beta=0,2, a_{0}=100 \cdot 10^{-6}, \tau=2$ и $N=19$. В отсчеты тестового сигнала добавлялась случайная аддитивная помеха, мощность которой составляла $50 \%$ от мощности сигнала. При этом оценки $s$ среднего квадратического отклонения (с.к.о.) по остаточной сумме квадратов были соизмеримы с оценкой с.к.о. $s=34,7 \cdot 10^{-6}$, вычисленной по результатам физического эксперимента. Исследовалась 1000 реализаций смоделированных таким образом функций. Одна из этих реализаций представлена на рис. 13 кривой 1 , а выборка ее значений зашумленных случайной помехой - точками 2. Графики восстановленных по этим точкам корреляционных функций представлены на рис. 13 кривыми 3 и 4. Кривая 3 построена по результатам вычисления параметров корреляционной функции с помощью итерационного численного метода среднеквадратичного оценивания коэффициентов ЛПДМ. Кривая 4 соответствует функции, параметры которой найдены с помощью численной процедуры решения нелинейной системы нормальных уравнений (с выбором первоначального приближения на основе результатов, полученных итерационным методом среднеквадратичного оценивания). Очевидно, что обе кривые практически совпадают и мало отличаются от истинной кривой 1 . Относительные погрешности определения параметров соответственно составили $\delta \alpha=56 \%, \delta \beta=0,8 \%$ и $\delta a_{0}=21 \%$ для первого случая и $\delta \alpha=42 \%, \delta \beta=1,0 \%$ и $\delta a_{0}=20 \%$ при втором методе вычислений. Остаточные суммы квадратов оказались равными $0,0171 \cdot 10^{-6}$ и $0,0167 \cdot 10^{-6}$, соответственно, что также свидетельствует о статистической эквивалентности результатов оценивания обоими методами. 
С целью сравнения погрешности вычисления параметров корреляционной функции в данном физическом эксперименте с предельными оценками относительной погрешности проведено усреднение относительных погрешностей вычисления параметров по 1000 реализаций: $\delta \alpha=85 \%, \delta \beta=4,8 \%$ и $\delta a_{0}=16 \%$. Видно, что эти значения в $2-3$ раза меньше предельно допустимых.

Таким образом, в работе получены следующие научные результаты:

- построена линейно параметрическая дискретная модель, описывающая последовательность отсчетов корреляционной функции распределения случайного поля неупругой реологической деформации;

- описан алгоритм определения параметров корреляционной функции на основе среднеквадратичных оценок коэффициентов линейно параметрической дискретной модели в форме стохастического разностного уравнения;

- разработан итерационный численный метод среднеквадратичного оценивания коэффициентов ЛПДМ и проведены численно-аналитические исследования его эффективности;

- разработана методика и получены формулы, описывающие предельные относительные погрешности вычисления параметров корреляционной функции;

- приведены результаты апробации итерационного метода среднеквадратичного оценивания в физическом эксперименте при исследовании ползучести материалов.

Все это позволяет говорить о том, что разработан новый эффективный метод определения параметров корреляционной функции распределения случайного поля неупругой реологической деформации, в основе которого лежит итерационная процедура среднеквадратичного оценивания коэффициентов линейно параметрической дискретной модели в форме стохастического разностного уравнения, и который может быть рекомендован для эффективного решения задач параметрической идентификации различных по физической природе объектов, динамические процессы в которых описываются затухающей по экспоненте синусоидой.

\section{БИБЛИОГРАФИЧЕСКИЙ СПИСОК}

1. Радченко В.П., Дудкин С.А., Тимофеев М.И. Экспериментальное исследование и анализ полей неупругих микрои макронеоднородностей сплава АД-1. // Вест. Самарского госуд. техн. ун-та. Сер. Физ.-мат. науки. 2002. Вып. 16. C. $111-117$.

2. Попов Н.Н., Самарин Ю.П. Исследование полей напряжений вблизи границы стохастически неоднородной полуплоскости при ползучести. // ПМТФ. 1988. №1. С. 159-164.

3. Должковой А.А., Попов Н.Н., Радченко В.П. Решение стохастической краевой задачи установившейся ползучести для толстостенной трубы методом малого параметра // ПМТФ. 2006. Т. 47. №1. С. 161 - 171.

4. Боглаев Ю.П. Вычислительная математика и программирование. М.: Высш. шк., 1990. 544 с.

5. Зотеев B.E. Разработка и исследование линейных дискретных моделей колебаний диссипативных систем. // Вест. Самарского госуд. техн. ун-та. Сер. Физ.-мат. науки. 1999. Вып. 7. С. 170-177.

6. Вучков И., Бояджсева Л., Солаков О. Прикладной линейный регрессионный анализ. М.: Финансы и статистика, 1987. $238 \mathrm{c}$

7. Грановский В.А., Сирая Т.Н. Методы обработки экспериментальных данных при измерениях. Л.: Энергоатомиздат. 1990.288 с. 